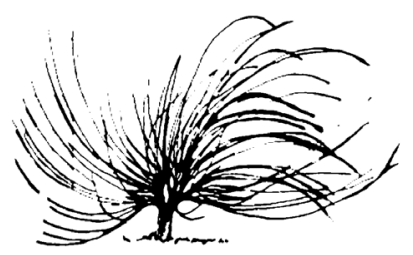

\title{
Desestructurando la mediación pedagógica tradicional: Experiencias y reflexiones de la propia praxis desde la estrategia de enseñanza de pareja pedagógica
}

\author{
Fiorella Araya Rodríguez ${ }^{l}$ \\ Centro Integral de Educación Privada \\ Costa Rica \\ fiorella.araya.rodriguez@una.ac.cr
}

\begin{abstract}
Resumen
Este ensayo corresponde a la praxis generada como estudiante universitaria, durante el programa de estudios de posgrado de la División de Educología, Universidad Nacional, entre enero 2018 y agosto 2019. Dentro de este mismo, se replante la mediación pedagógica tradicional al incluir una atractiva estrategia de enseñanza llamada pareja pedagógica, la cual sustenta la formación de profesionales en la Maestría en Educación con énfasis en Pedagogía Universitaria en una universidad pública de Costa Rica. Para argumentar esta propuesta, se acude al elemento autorreflexivo de la ensayista, así como a las referencias teóricas y al análisis de experiencias compartidas por parte de diferentes estudios que evidencian las fortalezas en torno a la construcción de nuevos saberes en el campo de la pedagogía costarricense en la educación superior.
\end{abstract}

\section{(c) (1) $(\Theta$}

http://dx.doi.org/10.15359/rep.esp-21.11

1 Diplomado en Inglés por la Universidad Nacional de Costa Rica (UNA), Bachiller en la Enseñanza del Inglés para I y II ciclos por la UNA, egresada de la Licenciatura en Enseñanza del Inglés para I y II ciclos de la Educación General Básica por la Universidad Hispanoamericana de Costa Rica (UH), actualmente estudiante de la Maestría en Educación con énfasis en Pedagogía Universitaria de la UNA, se desempeña como educadora y coordinadora del programa de Inglés de la institución educativa Centro Integral de Educación Privada (CIDEP). 
Palabras clave: Pareja pedagógica, mediación pedagógica, experiencias, praxis, reflexión.

\begin{abstract}
This essay corresponds to the praxis generated during the postgraduate studies' program of División de Educología, Universidad Nacional, from January, 2018 to August, 2019. As part of this essay, traditional pedagogical mediation is reconsidered by including an attractive teaching strategy known as co-teaching, which supports the training of professionals in the master's Program of Education with an emphasis on University Pedagogy at a public university in Costa Rica. To back up this proposal, the essayist turns to her self-reflection, as well as theoretical references and the analysis of shared experiences by different authors, which show the strengths around the construction of new knowledge in the field of Costa $\mathrm{Ri}-$ can pedagogy at the higher education level.
\end{abstract}

Keywords: co-teaching, pedagogical mediation, experiences, praxis, reflection.

\title{
Pensamientos introductorios
}

ngulo (2013) menciona que la educación se divide en dos extensiones que dan origen a las dimensiones de la racionalidad. 2 La primera inculca el deseo de explicar y construir los procesos educativos, y la segunda rescata justificar la validación de las acciones acontecidas durante el desarrollo de aprendizaje. Estas extensiones permiten que tanto la racionalidad de la representación, como la de la acción jueguen, un papel relevante en el entorno educativo.

Considerando estos rasgos de la razón y desde una posición formadora, encontramos que el proceso de enseñanza y aprendizaje se da mediante la búsqueda y la transformación de diversas estrategias que permitan un conocimiento más enriquecedor para los individuos a los cuales queremos facilitar dicho aprendizaje.

Exponen García y Jiménez (2018):

La formación pedagógica de los docentes universitarios se

ha convertido en una necesidad en nuestras universidades 
latinoamericanas. En el contexto costarricense, programas de posgrado de carácter profesionalizante y centrados en la pedagogía universitaria, buscan incentivar la reflexión sobre la propia praxis pedagógica con el propósito de trazar caminos hacia la transformación. (p. 2)

El pensamiento expuesto por las autoras García y Jiménez (2018) enfatiza gradualmente la relevancia en torno al tema de la transformación en las prácticas pedagógicas a nivel de posgrado en nuestro país. Se ha anunciado, frecuentemente, en diversos medios de comunicación, la urgencia de impulsar la educación en términos de mejora y calidad, y se pone en duda, además, de forma exhaustiva, el accionar docente dentro y fuera de las aulas'.

No obstante, es aquí cuando surge la inquietud de dar a conocer las estrategias que las universidades costarricenses están poniendo en práctica en el ámbito de las carreras en educación, con el objetivo de redireccionar la mediación pedagógica tradicional hacia un enfoque más auténtico y enriquecedor, tanto para los docentes como para el estudiantado, proponiendo, además, adaptaciones que logren saciar las carencias inmersas en el acto de enseñar, aprender e interactuar del contexto educativo.

Para ejemplificar, en el caso de la Universidad Nacional (UNA), universidad pública de Costa Rica, se oferta dentro del Sistema de Estudios de Posgrado, la Maestría en Educación con Énfasis en Pedagogía Universitaria, en la cual se contempla la formación de especialistas con entrega para generar de cambios que promuevan una sociedad desde una perspectiva más acorde con lo que se vive en el contexto de aula y praxis pedagógica, discentes con disposición a reflexionar y transformar, día tras día, las prácticas pedagógicas en el quehacer universitario.

Un aspecto notorio que destaca a este posgrado es la integración de la pareja pedagógica, la cual alude a que los sujetos docentes socialicen desde su propia praxis. En Latinoamérica, autores como Rodríguez

2 Constantemente se le atribuye un valor por separado a diversas áreas de estudio, dejando la educación como una actividad de índole recreativo. He aquí donde surgen estereotipos que denigran a más de una carrera, insultos tales como: "la maestra de preescolar sólo va a jugar con los niños", "el docente de inglés sólo va a poner canciones y a usar el diccionario", "para estudiar arte sólo hay que saber pintar". Pero, así como denigran, surgen también las opiniones que idolatran a aquellas otras carreras a quienes la sociedad les ha atribuido una connotación de mayor peso: "el doctor salva vidas", "el ingeniero diseña edificios", "el abogado lucha por la justicia”. 
(2014) y Suárez (2016) prefieren emplear el término de co-enseñanza para referirse a aquella colaboración conjunta entre profesionales capaces de combinar sus conocimientos para llegar a una reflexión práctica. Dicha acción reflexiva conlleva a un proceso analítico que remite a la pertinencia de valorar, redefinir y edificar ese intercambio de palabras y experiencias, con el fin de obtener una retroalimentación significativa, para poder continuar creando cambios para la construcción conjunta de nuevos saberes.

En palabras de Sanjurjo y Rodríguez (2005), "el docente desde el deber ser de su actuación profesional, como mediador y formador, necesita reflexionar sobre su práctica pedagógica para mejorarla y/o fortalecerla" (p. 37). Ser dos en un aula implica el trabajo cooperativo y la oportunidad de un acercamiento a un debate sano, pero crítico, de diversas cuestiones surgidas durante el recorrido pedagógico del par de profesionales.

Asimismo, a través de una comunicación asertiva entre sus principales participantes del proceso de educar y aprender, se posibilita la apertura de nuevas aproximaciones a la concientización y transparencia del proceso existente en la construcción de conocimientos. Los grupos docentes deben convertirse en docentes con ingenioso y creatividad, que apuesten por la implementación de un tipo de enseñanza diferente.

Es decir, una pareja pedagógica comprometida en dialogar y aprender mutuamente; más que educar autoritariamente, la nueva estrategia de enseñanza, propuesta por la Universidad Nacional cautiva tanto a actores como a espectadores para orientar un aprendizaje reflexivo, útil y oportuno para la comunidad universitaria.

Aunado a esto, el siguiente ensayo se encuentra orientado en generar una crítica constructiva de la estrategia en torno a las fortalezas presentes dentro del posgrado anteriormente mencionado. Desde la praxis universitaria, desde la mirada de la pareja pedagógica y su integración como una forma innovadora de participar, comprender y pertenecer en la pedagogía universitaria actual, buscamos que el estudiantado logre replantearse, con una visión más crítica y enriquecedora, el porqué del desestructuramiento que ha presentado la pedagogía tradicional. Podemos errar en nuestros pensamientos y reflexiones, pero estos juegos mentales de criticidad son los que nos han guiado hasta donde estamos. 


\section{El llamado a la desestructuración de la mediación pedagógica tradicional}

La pedagogía, al ser debatida entre dos fuertes oposiciones, origina una serie de reflexiones constantes de lo que realizamos en nuestras propias aulas como docentes. Por un lado, la pedagogía tradicional busca formar una ciudadanía que se adapte al orden establecido; y de esta manera la educación responda a las "demandas mercantiles del sector empresarial" (Martínez, 2014, p. 31). Por otro lado, existe la pedagogía crítica, que hace un llamado a la transformación de la educación en cuanto al replanteamiento de la realidad como tal, a través del diálogo crítico propuesto por Freire y Shor (2014), en su libro Miedo y osadía.

Al ahondar más sobre su existencia, procedemos a rescatar el término de mediación pedagógica, uno de los más utilizados dentro del contexto de praxis docente para dar referencia al acto del cómo y para qué educar. Parafraseando a Freire (1992), sostenemos que las vivencias y experiencias existenciales logran convertirse en mediación pedagógica, al provocar en los sujetos docentes reflexiones y análisis en torno a problemas, y permitirles, de esta manera, una verídica participación. Por consiguiente, la educación es comprendida como una mediación activa y transformadora del mundo, un diálogo reflexivo tanto sobre la acción como sobre la realidad misma. La práctica y los saberes de la experiencia de los sujetos son los puntos de partida -su aquí para llegar al allá-.

Sin duda alguna, la pedagogía ha sufrido transformaciones con el pasar de los años, sin embargo, es preocupante que aún hoy, continuemos mal gastando los esfuerzos invertidos por intentar mejorar el sistema educativo actual de nuestro país. Es lamentable el punto al que hemos llegado, al enfocarnos en proveer un aprendizaje significativo regulado por directrices y demandas escolares.

Al respecto, Martínez (2014) establece:

La instrucción es un proceso escolarizado en donde el nivel de rendimiento se fija a partir de la asimilación de un currículum y una asistencia obligatoria. Al final de cuentas, no importa quién aprende más, sino quién obedece las reglas que impone la institución escolar. La escuela no libera ni educa, porque las personas se vuelven menos autónomas y menos creativas. (p. 34) 
Con base en el pensamiento anterior, podemos resumir que, generalmente, la educación nos enseña a hablar, a leer y a escribir, y además nos dice lo que debemos hacer y no hacer, cuándo y qué pensar, el momento adecuado para hablar y callar y, ¿por qué no?, también nos enseñan las palabras por emplear. A eso le llaman educación. Nos encontramos en una constante lucha contra lo que queremos comunicar y no podemos, porque nos aterran las consecuencias que envuelven nuestras acciones, prefiriendo optar por un mutismo selectivo. Nosotros mismos encerramos nuestra esencia en una cárcel interna, una celda dentro de nuestro ser.

Es, en este sentido, por lo cual el modelo tradicional debe modificarse; no por su propósito de formar grupos de estudiantes con disciplina y rigidez en su rama de especialidad, sino por la inflexibilidad de preparar profesionales con disposición a pensar por sí, seres capaces de fundamentar sus ideologías con criticidad, pero, sobre todo, profesionales en pedagogía en la ruta de trabajar en conjunto para socializar nuevas propuestas educativas y transformadoras, partiendo de experiencias y reflexiones en relación con la propia praxis.

Así lo relatan Hernández y Flores (2012), al resaltar que un particular requisito de la mediación pedagógica sugiere la necesidad de partir de los conocimientos previos de quienes aprenden, lo cual involucra el conocer de la persona aprendiente en relación con la temática de estudio y las experiencias vividas.

En consecuencia, la pareja pedagógica viene a desestructurar la mediación pedagógica tradicional al intentar mejorar el accionar docente universitario mediante la constante vinculación entre práctica-teoría-práctica desde el área disciplinar; abre camino a una construcción compartida de saberes en pro de la transformación de la praxis pedagógica universitaria.

Es vital romper las barreras para dar paso a la transformación educativa. No se trata de leer y debatir rutinariamente, sino de sacar provecho y potenciar las opiniones críticas que existen ante diversos ejes temáticos por abordar en las aulas de posgrado con el estudiantado del siglo $\mathrm{XXI}^{3}$.

3 Ya no basta con una titulación que describa nuestra formación como profesionales del área, sino que, además, debe existir una vinculación más estrecha entre lo que enseñamos, aprendemos y la autorreflexión socializada; para formular respuestas de lo que está sucediendo en nuestro entorno y de esta manera, los saberes construidos puedan transformar la sociedad educativa. 
Desde este pensamiento podemos atribuir, a manera de cierre, que la mediación pedagógica requiere de constantes reestructuraciones para una óptima efectividad. Estas deben cumplir con las exigencias de una educación de calidad pensada para la formación de discentes del presente y futuro. Se ha vuelto una necesidad de cuidado el adaptar las herramientas y estrategias diseñadas ante una comunidad universitaria crítica y reflexiva, en torno a nuevas oportunidades de mejora en los procesos de enseñanza y aprendizaje.

Igualmente, la inclusión de una innovadora mediación pedagógica se convierte en aliada de la realidad del contexto de aula y de las experiencias vividas durante su trayecto. Este periodo de reflexión autocrítico puede ser también una etapa compartida con las personas que nos rodean en nuestro ambiente de trabajo, de esta manera resulta en un paradigma emancipador que cubre la colectividad de reflexiones, ideas, diálogo e intercambio de pensamiento; donde existe el apoyo de no solo una persona, sino de varios individuos que buscan transformar y crear nuevas perspectivas dentro del área de la pedagogía.

El proceso de experimentar, vivir y respirar la pedagogía desde la práctica le facilita al cuerpo docente y estudiantil progresos de desarrollo en su especialidad, así como la visión de un panorama más certero, en términos de realidad. Constantemente, nos hacemos el mismo cuestionamiento todos los días: ¿qué se debe priorizar en la educación costarricense? Es precisamente en este punto cuando surge la diferencia de una pedagogía tradicional y una pedagogía crítica. No se trata de evidenciar la categorización en un pedazo de papel, sino de llevar la transformación a la práctica con el fin de obtener nuevas mejoras en el campo de la educación.

Freire (1997) expresa que "nadie educa a nadie - nadie se educa a sí mismo - los hombres se educan entre sí mediatizados por el mundo" (p. 73). Ello concierne, que, al socializar, es indispensable aclarar que no se quiere imponer un pensamiento o una experiencia sobre otra, sino de originar la apertura de un diálogo dinámico, equitativo y transformador. Un diálogo que nos acerque a la pedagogía y no que nos divorcie de esta misma; esto es lo que se entiende como desestructuramiento en la mediación pedagógica tradicional a través de la pareja pedagógica. 


\section{Conceptualización de la estrategia de enseñanza de pareja pedagó- gica: ¿Qué? ¿Cómo? ¿Por qué?}

Quienes accionamos en nuestra labor diaria como facilitadores en los entornos práctico-pedagógicos de la educación superior, realizamos inagotables intentos por facilitar los saberes, partiendo de "tres componentes primordiales: (a) los docentes, (b) los alumnos, y (c) el proceso formativo" (Díaz, 2006, p. 90).

Esta entidad formadora en la maestría en Educación con énfasis en Pedagogía Universitaria, posgrado del cual se ha originado este escrito, suscita la relevancia del pensamiento reflexivo, ontológico, epistemológico y teórico entre los procesos pedagógicos del saber y el hacer, permitiendo, además, el origen de interrogantes que cuestionan críticamente la praxis y las múltiples facetas para concebir el conocimiento. Entre las cuales, deslumbra la estrategia de enseñanza de pareja pedagógica y su impacto transformador.

Debido a lo supra mencionado, debemos desarrollar las siguientes interrogantes, ¿qué entendemos por pareja pedagógica? ¿cómo se integra la pareja pedagógica en la educación superior? y, ¿por qué debemos continuar ejerciendo dicha estrategia en las clases universitarias?

Para comenzar, parafraseando a Rodríguez (2014), comprendemos la pareja pedagógica como la co-enseñanza de dos personas que comparten la responsabilidad de un grupo de estudiantes, y del cual este par de profesionales promueven la ayuda colaborativa para abarcar las necesidades del estudiantado. Por otra parte, García y Sánchez (2018), conceptualizan la pareja pedagógica como "un dispositivo de formación en la que dos docentes reflexionan sobre su praxis pedagógica; para ello recurren a la observación de sus actuaciones en clase y el análisis de lo ocurrido a partir del diálogo con su par" (p. 4).

Añadido a esto, Beltramo (2012) manifiesta que este dispositivo de formación es considerado como un acompañamiento realizado entre pares al momento de desarrollar una unidad didáctica; en donde dicha compañía favorece la expresión reflexiva sobre la praxis pedagógica, así como el enfrentamiento de diversos hechos, sentimientos y emociones que surgen en los procesos educativos, sean estos positivos o negativos.

En razón de lo definido por estos autores, redireccionamos el término de pareja pedagógica como la gestión de un entorno colaborativo entre dos personas pedagogas y su compromiso por entablar un proceso 
de co-enseñanza y aprendizaje, que abren camino a la reflexión de la propia praxis en la mediación pedagógica.

Ahora bien, al hablar de la integración del dispositivo de pareja pedagógica como estrategia generadora de saberes en la educación superior, es indispensable recalcar que, para efectos educativos, se requiere de dos sujetos colaboradores activos e interesados por mejorar las prácticas docentes. Desde esta perspectiva, la integración de la práctica surge cuando ambas partes aportan conocimiento, visualizan las posibles amenazas de trabajo y resaltan sugerencias de oportunidades de mejora a través de diálogos interactivos, asertivos y oportunos.

Como exponen Sanjurjo et al. (2016):

Analizar las tareas realizadas en el aula, escuchar la perspectiva de un par que está compartiendo la misma experiencia ayuda a modificar actitudes y a tomar decisiones más ajustadas en el complejo proceso de hacer la clase. Específicamente, los residentes destacan y valoran las intervenciones del par en cuanto a cómo se desarrolla la exposición dialogada, se realiza el manejo de los tiempos, el uso del vocabulario, del pizarrón, de otros recursos tecnológicos, el tratamiento de lo grupal. Este trabajo de intercambio, análisis, interpretación, reflexión sistemática en la pareja pedagógica estimula y genera confianza y la posibilidad de replantear la propia práctica. Es decir, el trabajo en pareja pedagógica como evaluación formativa, constituye una suerte de "efecto espejo". (p. 9)

El "efecto espejo" que retratan Sanjurjo et al. (2016) sustentan ese aprendizaje mutuo y profusamente beneficioso para el crecimiento y la transformación profesional de los actores directos; pues, ese aprendizaje es reflejo de lo que logramos observar, enseñar y aprender entre pares. En este sentido, únicamente el par de docentes son quienes poseen la dicha de experimentar y validar un diálogo constructivista e igualitario; reluciendo las fortalezas y debilidades de las prácticas docentes.

Expuesto lo anterior, podemos avanzar a responder el tercer y último interrogante con respecto al por qué debemos continuar ejerciendo dicha estrategia en las clases de educación superior.

Para ello, Salamanca (2011) relata en su texto Dos docentes, dos miradas: La pareja educativa reflexiones retroalimentativas en 
consideración a la formación docente colaborativa entre pares, así como virtudes que renueven esta profesión. Dentro de las ventajas propuestas por Salamanca (2011) se rescatan, primordialmente, la fragmentación del aislamiento y la mejora de la propia praxis docente.

Por una parte, la tarea de educar es compartida a manera tal que la vivencia conjunta de dos profesionales apoyándose hombro con hombro, postura con postura e ideología con ideología, repercuta en el incremento del deseo por verse crecer mutuamente. Se generan debates y conflictos, pero todo eso es parte de ese diálogo transparente y enriquecedor del que hemos venido conversando.

Por otro lado, la reflexión se desprende de este último punto, donde la práctica pide que se haga una valoración personal sobre la experiencia y los aspectos que se pueden mejorar en el proceso de co-enseñanza-aprendizaje ${ }^{4}$. Al entrar en la etapa reflexiva de forma asertiva, somos capaces de socializar, no para perjudicar sino para mejorar el cómo abordamos los contenidos y las inquietudes del estudiantado en nuestro quehacer educativo.

En la actualidad, nos encontramos en una paradoja cuando optamos por transformar, pues inevitablemente partimos de un miedo a la crítica destructivas y a las resistencias, más la unificación entre determinación y el empoderamiento por buscar nuevas maneras de hacer, dan como resultado la reflexión entre lo que hacemos y lo que somos capaces de hacer.

Ciertamente, el dispositivo de la pareja pedagógica resulta alentador para quienes, con curiosidad descubrimos nuevas huellas en los senderos formativos dentro de la comunidad universitaria costarricense, posibilitando aquellas y otras virtudes que respondan la diligencia de continuar ejerciendo la estrategia de co-enseñanza. Las transformaciones son buenas siempre y cuando sean fortalecidas con el vivir y el experimentar a lo largo de sus integraciones.

Es aquí donde creemos que la pareja pedagógica convierte los entornos de aprendizaje en ambientes de construcción, desconstrucción

4 Socializar nuestra identidad profesional entre colegas, amplía el panorama de quienes somos y para dónde vamos La co-enseñanza-aprendizaje nos hace madurar en este proceso y eso provoca que, al aceptarnos los puntos de vista de mejora, podemos dar paso a un avance profesional de nuestro rol como agentes del cambio en las aulas.

5 Es evidente que, en ocasiones, los docentes experimentemos situaciones que invaden y destruyen nuestra labor como formadores. No obstante, debemos aprender a pensar la reflexión intrínseca de nuestra praxis pedagógica como un ejercicio no de destrucción, sino de aprendizaje. 
y reconstrucción en el proceso reflexivo; en una "forma de practicar la crítica con el objetivo de provocar la emancipación de las personas, cuando descubren que tanto el saber pedagógico como la práctica pedagógica son construcciones sociales de la realidad"'(Gimeno y Pérez, 2000, p. 419).

\section{Transformaciones intrínsecas: Vivencias inmersas en la práctica pedagógica}

Desde la propia experiencia, como estudiante y educadora, podemos llegar a pensar sobre dos importantes posturas de ejercicio de la pedagogía existentes en Costa Rica. En primera instancia, se encuentran los grupos de docentes que se inclinan por replicar los trabajos antecesores. Profesorado aterrado por el cambio y asumen por un hecho que las medidas tomadas hasta el momento son las idóneas. Son amantes de una conocida rutina, que además busca la producción de conocimientos validados a través de una prueba escrita', esos grupos docentes obstaculizan el proceso y cumplen únicamente con lo establecido en el currículo escrito.

En contraste, existen quienes ejercen una pedagogía apasionada, en la que me inscribo. Nos dedicamos a nuestras clases y planeaciones. Somos docentes con compromiso por innovar, con el objetivo de quebrantar impedimentos para obtener mejores resultados. Somos educadores y educadoras que anhelamos una transformación más allá del cumplimiento de los contenidos. Queremos abarcar las necesidades educativas del país al indagar, estudiar, analizar y reflexionar sobre lo que realmente necesita el sistema educativo.

Para este caso en particular, la maestría en Educación con énfasis en Pedagogía Universitaria de la Universidad Nacional, al poseer una modalidad semipresencial con apoyo virtual, alude a que el estudiantado se responsabilice de su propio proceso de enseñanza aprendizaje y pueda, adicionalmente, hacer uso de recursos que potencien tanto la creatividad como la criticidad con las lecturas y aportes generados por parte de sus docentes y los compañeros y compañeras de cada uno de los módulos. Partiendo de esta estructuración tan rica en cuanto a

6 La rutina nos destruye porque impide que innovemos en el sistema educativo. Nos impide, a nosotros los educadores, a presenciar más allá de lo que se aprende en un libro. Es pertinente el poder darle hincapié a una enseñanza más experimental y útil en contraposición a la aprobación de un examen. 
contenido curricular, ¿de qué manera ha favorecido la inclusión de la pareja pedagógica a la población estudiantil del programa de posgrado?

Para responder dicha interrogante, la experiencia adquirida a lo largo de la duración del programa de posgrado ha permitido corroborar cómo la dinámica de trabajo entre dos docentes dentro del aula, en sus roles facilitadores de los aprendizajes, compensa gradualmente el desempeño del alumnado en relación con su desarrollo profesional. $\mathrm{Al}$ estar en un ambiente de clase que promueve un aprendizaje compartido, constructivo, pero sobre todo reflexivo, crea una motivación que incentiva a que tanto docentes como estudiantes añoren con seguir aprendiendo entre sí.

La utilidad de la pareja pedagógica simboliza ese compromiso por dar colaboraciones auténticas en comparación con los aportes impregnados únicamente de forma textual o de manera individualista entre docentes y aprendices; se convierte en una novedosa tendencia que reemplaza los antiguos métodos de enseñanza tradicionalistas que anulan la participación activa del estudiantado como rol esencial en el proceso de aprendizaje, porque "la pedagogía, o al menos la mediación pedagógica es, ante todo, un acto de amor que va más allá de lo disciplinar, pues al ser su 'objeto' los procesos de enseñanza y aprendizaje, implica a las personas mismas" (Gómez, 2016, p. 5).

Es trágico siquiera pensar en aquel profesorado que transforma de manera individualizada; o peor aún, la existencia de un empoderado celo académico entre docentes ${ }^{7}$, lo cual no da cabida a una apertura al diálogo e intercambio de saberes, anulando, por completo, la criticidad y la reflexión sobre la práctica entre colegas.

Este dispositivo de enseñanza revoluciona la condición individualista en la que la comunidad universitaria ha estado inmersa junto con las condiciones dogmáticas y conductistas con las que ha convivido. El poseer dos docentes a cargo de un módulo, en educación superior, enriquece el manejo grupal, pues al entablar un espacio de construcción conjunta de conocimientos, la versatilidad de la concentración y el respaldo de dos personas expertas genera sanos debates entre aprendices: "dispositivo pareja pedagógica propicia la transición de un sujeto en

7 Así también, como la constante transferencia de responsabilidades: "no es mí culpa, es suya". Un colectivo de docentes debe de acuerparse y velar por el cumplimiento de una preparación de calidad. Ya basta con señalarnos unos a los otros, es necesario actuar; porque al final del día es lo que el contexto educativo necesita: más soluciones. 
formación a un profesional auto dirigido y de una cultura individualista a una comunidad de aprendizaje" (Rodríguez y Grilli, 2013, p. 22).

Demás está decir que la pertinencia de un replanteamiento de a quién involucra el acto de educar, de quienes aprendemos y a quienes enseñamos. Se refiere, entonces, a la práctica insaciable de reflexionar la singularidad de cada sujeto; y de cómo sus peculiaridades influyen en la generación colectiva de conocimientos teóricos y prácticos. No se busca someter una posición sobre otra; sino por el contrario, aprender una de otra, motivando a seguir creciendo como seres humanos íntegros y de mucho saber.

Como sociedad, cada individuo desarrolla una personalidad distinta y al intentar enseñar a pensar, reflejamos un mundo de respuestas infinitas. Siendo educadores y educadoras, alentamos al estudiantado a formular un criterio en relación con los temas por socializar.

Aunado a esto, replica De Luca (2009):

En la medida en que nos evaluamos y conocemos, nos acercamos más al autoconocimiento, percatándonos de la libertad que disponemos o de la capacidad que poseemos para tomar decisiones libres, para dialogar e intercambiar ideas, con un estilo propio, maximizando el potencial analítico y creativo con el que contamos, fortaleciendo los valores y principios sociales a los que responde el comportamiento. (p. 909)

El sistema de educación superior alienta al estudiantado a ser formado como profesionales; es por ello que la comunicación asertiva y nuestra propia aceptación, no solo como estudiantes o docentes, sino también como seres humanos, es la salida a los múltiples conflictos que atravesamos rutinariamente.

En coherencia con el desarrollo de la criticidad y el autoconocimiento, podríamos indicar que la pareja pedagógica logra acentuar el accionar en la educación como una etapa integral que aproxima al sujeto a aprender no solo de su propia realidad, sino de las realidades contextuales de quienes lo rodean ${ }^{8}$; promoviendo la apertura a la propuesta de alternativas. Hablamos, además, de la inclusión de una mediación pedagógica que se apegue a las experiencias vividas en el

8 Cada individuo posee una historia, tal vez incluso no contada. No se ve el esfuerzo, las derrotas y lo vivenciado. Tendemos a enfocarnos en los logros como meta primordial. 
proceso; y a partir de allí, crear reflexiones autocríticas de lo que implica la pedagogía.

Partiendo de la socialización, no nos encontramos en un diálogo de sordos. Al entablar una productiva conversación, más que una defensa, somos capaces de escuchar cada voz, opinión y sugerencia para poder llegar a una negociación justa en el diálogo. Respecto a esto, Calderón (2001), expone que "aulas abiertas al diálogo y a la participación de todos propician un ambiente de aprendizaje apto para la construcción del conocimiento" (p. 137).

De esta manera, quienes hemos vivenciado el trabajo de la pareja pedagógica desde una postura de crecimiento profesional, logramos concebir la pedagogía como un ente regulador entre las enseñanzas conjuntas de cada participante, transformando la educación en un proceso de aprendizaje mutuo, una evaluación continua de lo que deberíamos estar haciendo y lo que en realidad hacemos: acercarnos aún más al cambio.

\section{Conclusiones y recomendaciones pedagógicas}

A este punto, es gratificante mencionar que la Maestría en Educación con Énfasis en Pedagogía Universitaria de la UNA inspira la formación de especialistas con entrega en generar cambios que promuevan una sociedad desde una perspectiva más acorde con lo que se vive en el contexto de aula y praxis pedagógica. En este ensayo, hemos socializado dos puntos claves: la desestructuración de la mediación pedagógica tradicional, y la estrategia de pareja pedagógica a nivel de educación superior, para la apertura de experiencias y reflexiones de la propia praxis.

Por un lado, al referirnos a la desestructuración en la mediación pedagógica tradicional, pensamos en un nuevo camino por recorrer, un camino que permita igualdad de oportunidades entre la teoría, la praxis y la reflexión. No se trata de darle mayor importancia a una parte u a otra, ni de promover un menor uso o un abuso de estas. Lo que se quiere es que, de manera conjunta, puedan impregnarse para que los sujetos involucrados sean quienes dirijan su propio modelo de enseñanza y aprendizaje a partir de lo aprendido y vivenciado por equipos reconocidos exponentes de la pedagogía, por las experiencias tejidas en la clase, por lo aprendido con los compañeros y compañeras del módulo y por las experiencias compartidas a cargo de la pareja pedagógica. 
De este modo, sus participantes aprenderán mientras practican y viven la pedagogía, porque, "en estos espacios de socialización entre colegas de diferentes instituciones educativas, las cuales tienen sus propias historias, rutinas y su cultura, es donde pueden poner en crisis sus perspectivas y trascender hasta la reflexión" (Contreras 1999).

Por otro lado, como bien se ha mencionado, la pareja pedagógica busca reflexionar sobre las acciones cometidas, promoviendo un pensamiento crítico reflexivo de los entornos educativos, a fin de encontrar soluciones para futuros eventos. Al desarrollar la pedagogía desde la estrategia de co-enseñanza, implica ubicarnos no solo desde una posición individualista, sino también desde el contexto del otro ser.

Un pedagogo o pedagoga que es capaz de reflexionar desde una praxis dinámica y compartida crece de manera profesional, puesto que crea aprendizajes que le llevan a repensar nuevas metodologías de enseñanza, nutriéndose de nuevos conocimientos. Esto propicia un aprendizaje que pueda ser socializado entre quienes anhelan seguir no solo investigando la pedagogía, sino también aplicarla con el objetivo de alcanzar nuevos avances en ella y la comunidad educativa, para, de esta manera, contribuir a la concepción y práctica de la pedagogía como un entorno de vivencia y transformación. Expuesto lo anterior, procedemos a resumir cinco recomendaciones que procuran concientizar al público lector para continuar aprendiendo de la propia praxis.

La primera sugerencia, propone continuar empleando la estrategia de pareja pedagógica entre profesorado universitario. Desde la propia experiencia, se ha discutido a lo largo del ensayo cómo el haber cursado la maestría a cargo de dos personas pedagogas en los cinco módulos, facilitó la puesta de conocimiento, así como la fluida dialogicidad vivenciada entre cada participante. Esto conlleva a potenciar la formación profesional de los futuros cuerpos docentes, partiendo de diversos contextos, posiciones e ideas socializadas que poco a poco ocasionan la desestructuración de la mediación pedagógica tradicional.

La segunda recomendación sugiere capacitar al personal docente sobre oportunidades de mejora en torno a la propia praxis. Es importante reconocer que, como personal pedagogo universitario, nos encontramos en un ambiente de constante cambio; en donde nuevas estrategias educativas surgen y renuevan nuestro quehacer diario. Así lo enfatiza Zapico (2019): "cada persona debe hacer su aporte a conciencia desde su área de trabajo. Docentes deben revisar sus prácticas 
y actualizarse a fines de utilizar información errónea" (p. 30). En este sentido, la capacitación nos impulsan a aprender nuevamente sobre cómo progresar de manera asertiva en las prácticas pedagógicas, así como la puesta en común de innovadoras estrategias tanto de enseñanza como de aprendizaje.

En tercera instancia, se recomienda integrar la estrategia de pareja pedagógica en asignaciones evaluativas, de índole formativo y sumativo, orientadas hacia el estudiantado. Involucrar a los sujetos aprendices a participar de esta estrategia de co-enseñanza; les invita a observar, analizar y exponer situaciones en contextos reales, para eventualmente, aportar apreciaciones en relación con la mediación pedagógica experimentada, porque "las conversaciones valiosas para la adquisición de nuevo conocimiento ocurren con la realidad, con otras personas y con uno" (Carbonell, 2015, p. 223).

La cuarta recomendación, hace referencia a una posible expansión de la estrategia de pareja pedagógica en los diferentes niveles del sistema educativo costarricense: preescolar, primaria, secundaria y educación superior. Esta propuesta invita a que, desde la etapa inicial de estudio, el estudiantado pueda devolverse en un espacio de formación académica, con pensamientos más amplios y críticos, construidos entre pares, cuyas bases de los saberes son facilitadas por dos profesionales capaces de aportar, sin la necesidad de juzgar las prácticas docentes contrarias.

Finalmente, invitamos a la socialización de experiencias entre colegas y estudiantes en relación con la puesta en práctica de la estrategia de pareja pedagógica. Indica Aguilar (2015) que "hay que partir de la realidad, reflexionar esa realidad y volver a la realidad practicando, corrigiendo las fallas que se han descubierto en esa reflexión" (p. 23). Desde el accionar en la educación, la libre socialización ayuda a la transformación pedagógica para mejorar la mediación en las clases, lo cual da, como resultado, el crecimiento de seres humanos sensibles, críticos, y profesionales de la pedagogía. 


\section{Referencias}

Aguilar, C. (2015). Paulo Freire: El camino de la praxis pedagógica al inédito viable. Pontifica Universidad Javeriana.

Angulo, F. (2013). Breve introducción a la epistemología en educación. Universidad de Cádiz, España. https://www.academia. edu/5617561/Epistemolog\%C3\%ADa-Educaci\%C3\%B3n

Beltramo, L. (2012). ¿Cómo opera la pareja pedagógica como dispositivo de formación docente? Revista de la Escuela de Ciencias de la Educación, 7, 255-266.

Calderón, K. (2001). La didáctica hoy. Las concepciones y aplicaciones. San José, EUNED.

Carbonell, J. (2015). Pedagogías del siglo XXI: Alternativas para la innovación educativa. Octaedro.

Contreras, J. (1999). La autonomía del profesorado. Ediciones Narcea.

De Luca, C. (2009). Implicaciones de la formación en la autonomía del estudiante universitario. Electronic Journal of Research in Educational Psychology, 7(18), 901-922.

Díaz, V. (2006). Formación docente, práctica pedagógica y saber pedagógico. Revista de Educación Laurus: Venezuela, 12, 88-103.

Freire, P. (1992). Pedagogia da esperança: um reencontro com a pedagogia do oprimido. Paz e Terra.

Freire, P. (1997). Pedagogia de la autonomía. Saberes necesarios para la práctica educativa. Siglo XXI.

Freire, P. y Shor, I. (2014). Miedo y osadía. La cotidianidad del docente que se arriesga a practicar una pedagogía transformadora. Siglo Veintiuno.

García, M. y Jiménez, S. (2018). La experiencia del trabajo en pareja pedagógica entre docentes universitarios de diferentes disciplinas: Un dispositivo para la reflexión de la propia praxis. Ponencia presentada en el $X$ Congreso Iberoamericano de Docência Universitária, Porto Alegre, Brasil.

Gimeno J. y Pérez A. (2000). Comprender y transformar la enseñanza. Morata.

Gómez, J. (2016). Pedagogía, una disciplina indisciplinada: Límites de un debate y sus perspectivas ético políticas. Revista Electrónica Educare, 20(3), 1-12. 
Hernández, A. y Flores, L. (2012). Mediación pedagógica para la autonomía en la formación docente. Revista Electrónica Educare, 16(3), 37-48. http://www.revistas.una.ac.cr/index.php/ EDUCARE/issue/current

Martínez, R. (2014). Pedagogía tradicional y pedagogía crítica. CELAPEC.

Rodríguez, E. y Grilli, J. (2013). La pareja pedagógica: Una estrategia para transitar y aprender el oficio de ser profesor. Páginas de Educación, 6(1), 61-81. http://www.scielo.edu.uy/scielo.php?script=sci_arttext\&pid=S1688-74682013000100004\&lng=es\&tlng=es.

Rodríguez, F. (2014). La co-enseñanza, una estrategia para el mejoramiento educativo y la inclusión. Revista Latinoamericana de Educación Inclusiva, 8(2), pp.219-233. http://www.rinace.net/ rlei/numeros/vol8-num2/art11.pdf

Salamanca, C. (2011). Dos docentes, dos miradas: La pareja educativa. Revista de Investigación e Innovación Educativa, 42, 95-102.

Sanjurjo, L. y Rodríguez, J. (2005). Volver a pensar la clase: Las formas básicas de enseñar. Homo Sapiens Ediciones.

Sanjurjo, L., Caporossi, A. y Placci, N. (2016). La pareja pedagógica como dispositivo de evaluación en la formación docente universitaria. Ponencia presentada en Jornadas sobre las Prácticas de Enseñanza en la Formación Docente, Universidad de Quilmes, Bernal, Argentina. http://ridaa.unq.edu.ar/handle/20.500.11807/764

Suárez, G. (2016). Co-enseñanza: Concepciones y prácticas en profesores de una Facultad de Educación en Perú. Revista Electrónica de Investigación Educativa, 18(1), 166-182. http://redie.uabc. $\mathrm{mx} /$ redie/article/view/786

Zapico, M. (2009). ¿Por qué enseñar abstracciones en la educación primaria y secundaria? Evidencia contra las corrientes actuales. Revista Ensayos Pedagógicos, 14(1), 17-35. https://doi. org/10.15359/rep.14-1.1 